\title{
Recent Results and Running Experience of the New Aleph Vertex Detector
}

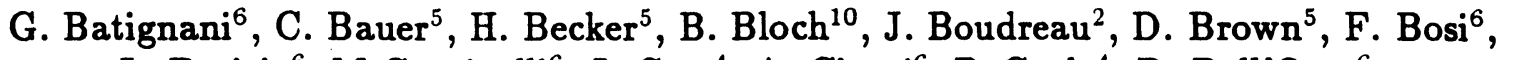

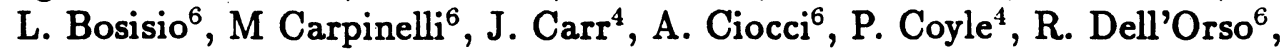
H. Dietl ${ }^{5}$, J. Drinkard ${ }^{4}$, E. Focardi ${ }^{6}$, F. Forti ${ }^{6}$, M. Giorgi ${ }^{6}$, T. Hansl-Kozanecka ${ }^{5}$, D. Hauff ${ }^{5}$, P. Holl ${ }^{5}$, R. Jacobsen ${ }^{2}$, E. Lancon ${ }^{2}$, J. Lauber ${ }^{5}$, A. Litke ${ }^{7}$, G. Lutz ${ }^{5}$, G. Lütjens ${ }^{5}$, E. Mannelli ${ }^{6}$, W. Männer ${ }^{5}$, T. Mattison ${ }^{2}$, M. McNeal ${ }^{7}$, S. Menary ${ }^{2}$, L. Moneta ${ }^{1}$, H.G. Moser ${ }^{2}$, B. Mours ${ }^{11}$, G. Parrini ${ }^{1}$, S. Piccinini ${ }^{6}$,

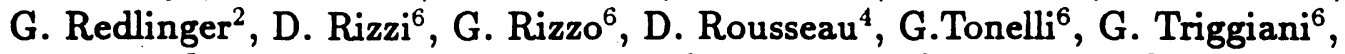
A.S. Schwarz ${ }^{5}$, P. Schwemling ${ }^{4}$, R. Settles ${ }^{5}$, H. Seywerd ${ }^{8}$, V. Sharma ${ }^{9}$, L. Strüder ${ }^{5}$,

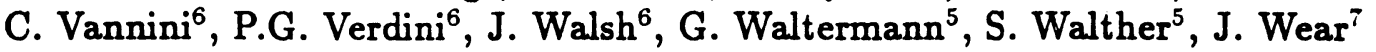
and F. Weber ${ }^{9}$.

Presented by Guido Tonelli INFN and University of Pisa, Italy.

1) University and INFN, Florence, Italy; 2) CERN, Geneva, Switzerland;

3) University of Mainz, Germany; 4) Centre de Physique de Particules, Marseille, France;

5) MPI, Munich, Germany; 6) University, INFN and Scuola Normale Superiore, Pisa, Italy;

7) University of California, Santa Cruz,USA; 8) University of Siegen, Germany;

9) University of Wisconsin, Madison, USA; 10) CEN, Saclay, France; 11) LAPP, Annecy, France.

\begin{abstract}
A short description of the new Aleph Vertex Detector (VDET 91), including the new mechanical structure and the improved DAQ system is given. The apparatus consists of two complete layers of silicon detectors with doublesided readout which provide a full $x, y, z$ information of the impact points of charged particles. The paper shows results on signal to noise ratio for minimum ionizing particles (m.i.p.) and efficiency in hit/track matching. Results on position, impact parameter and momentum resolution are also shown as measured using high momentum muons. Finally the first preliminary study on vertexing techniques and their impact on physics analysis are discussed.
\end{abstract}

Paper presented at the 1991 IEEE Nuclear Science Symposium, November 2-9, 1991, Santa Fe, New Mexico, USA.

\section{UNIVERSITÃ DEGLI STUDI DI PISA}




\title{
Recent Results and Running Experience of the New Aleph Vertex Detector
}

\author{
G. Batignani ${ }^{6}$, C. Bauer ${ }^{5}$, H. Becker ${ }^{5}$, B. Bloch ${ }^{10}$, J. Boudreau ${ }^{2}$, D. Brown ${ }^{5}$, F. Bosi ${ }^{6}$,

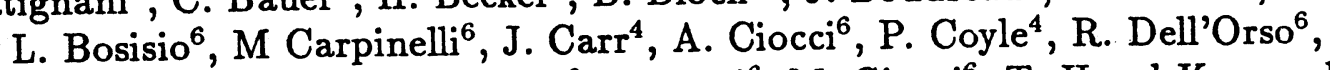 \\ H. Dietl ${ }^{5}$, J. Drinkard ${ }^{4}$, E. Focardi ${ }^{6}$, F. Forti ${ }^{6}$, M. Giorgi ${ }^{6}$, T. Hansl-Kozanecka ${ }^{5}$,

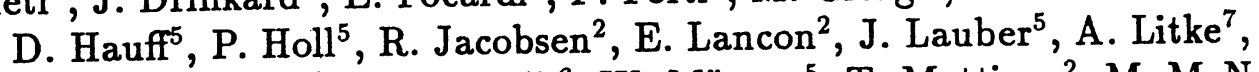 \\ G. Lutz ${ }^{5}$, G. Lütjens ${ }^{5}$, E. Mannelli ${ }^{6}$, W. Männer ${ }^{5}$, T. Mattison ${ }^{2}$, M. McNeal ${ }^{7}$, \\ S. Menary ${ }^{2}$, L. Moneta ${ }^{1}$, H.G. Moser ${ }^{2}$, B. Mours ${ }^{11}$, G. Parrini ${ }^{1}$, S. Piccinini ${ }^{6}$,

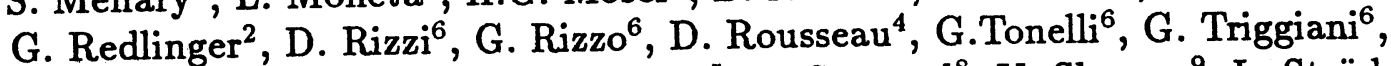 \\ A.S. Schwarz ${ }^{5}$, P. Schwemling ${ }^{4}$, R. Settles ${ }^{5}$, H. Seywerd ${ }^{8}$, V. Sharma ${ }^{9}$, L. Strüder ${ }^{5}$,

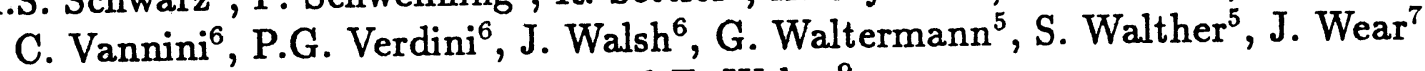 \\ and F. Weber ${ }^{9}$. \\ Presented by Guido Tonelli \\ INFN and University of Pisa, Italy.
}

1) University and INFN, Florence, Italy; 2) CERN, Geneva, Switzerland;

3) University of Mainz, Germany; 4) Centre de Physique de Particules, Marseille, France;

5) MPI, Munich, Germany; 6) University, INFN and Scuola Normale Superiore, Pisa, Italy;

7) University of California, Santa Cruz,USA; 8) University of Siegen, Germany;

9) University of Wisconsin, Madison, USA; 10) CEN, Saclay, France; 11) LAPP, Annecy, France.

\begin{abstract}
A short description of the new Aleph Vertex Detector (VDET 91), including the new mechanical structure and the improved DAQ system is given. The apparatus consists of two complete layers of silicon detectors with doublesided readout which provide a full $x, y, z$ information of the impact points of charged particles. The paper shows results on signal to noise ratio for minimum ionizing particles (m.i.p.) and efficiency in hit/track matching. Results on position, impact parameter and momentum resolution are also shown as measured using high momentum muons. Finally the first preliminary study on vertexing techniques and their impact on physics analysis are discussed.
\end{abstract}

\section{INTRODUCTION}

At LEP energy, namely sitting on the $Z^{0}$ peak, the large coupling to all fermion states gives a sizeable amount of $\tau$ lepton pairs and of heavy quark jets. The study of production and decay properties of these short-lived particles can be significantly improved if the tracking accuracy is good enough to deal with average transverse impact parameters of the order of $100 \mu \mathrm{m}$ and secondary vertices a few $\mathrm{mm}$ apart in space. This is the main goal of the Aleph Vertex Detector (VDET).

The apparatus consists of two layers of double-sided microstrip silicon detectors that measure the impact points of high energy ionizing particles in both $r-\phi, r-z$ coordinates.

A first version of this detector was installed in spring 1990 and took data during the 1990 LEP run[1].

At the end of the run a new beryllium beam pipe with a smaller outer radius $(5.5 \mathrm{~cm})$ was installed in Aleph[2]. A completely new vertex detector (VDET91) has been built and installed in spring 1991[3]. The main changes with respect to the earlier version are: 1) the equipment of two complete layers; 2) a new mechanics accounting for the smaller beam pipe and a different installation scheme; 3 ) the use of a new, improved digitizer in the DAQ; 4) a better protection scheme for the power supply of the detectors.

This paper describes the most recent results obtained with this new detector, the main problems faced in the long running period and the first clues of physics potential of high precision $3-\mathrm{D}$ vertexing.

\section{General Layout}

VDET91 consists of two concentric barrels of double-sided Si microstrip detectors positioned around the interaction region close to the beam pipe. The inner layer houses 9 elementary detector units (called 'faces') at a radius of approximately $6.3 \mathrm{~cm}$ from the beam; 15 'faces' are mounted on the outer layer at an average radial distance of about $10.7 \mathrm{~cm}$. The 'faces' are arranged in such a way as 
to allow full azimuthal coverage. The 0 coverage is $\pm 58^{0}$ for the inner layer and $\pm 44^{\circ}$ for the outer layer. An overall $82 \%$ of the solid angle is covered by the inner layer ( $74 \%$ by the two layers). A sizeable amount of tracks (5\%) pass through the overlap regions between adjacent 'faces' to allow a better alignment.

\section{The Silicon Detectors}

Each 'face' consists of two independent 'modules' assembled together. Each 'module' houses two double-sided microstrip detectors $51.2 \times 51.2 \mathrm{~mm}^{2}$ for a total number of 96 silicon wafers [fig.1].

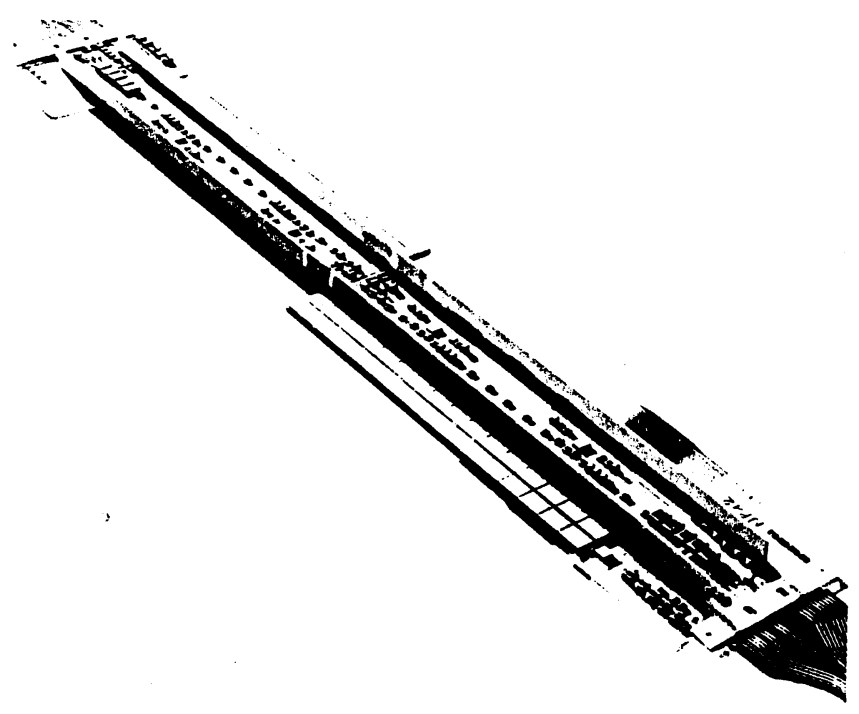

Fig.1 $Z$-side view of one 'face'

Each detector used for the construction of VDET91 has been carefully tested in order to meet very stringent selection criteria for high quality devices. Details of this procedure and of the technology of double-sided silicon detectors can be found elsewhere $[4,5]$.

The junction side of each detector is used to measure the $r-\phi$ coordinate whilst the $z$ coordinate is measured by the strips on the ohmic side. On both sides the strip pitch is $25 \mu \mathrm{m}$ and read-out pitch $100 \mu \mathrm{m}$. The number of read-out strips on each detector is 994 ( 497 for each side). The $r-\phi$ strips of two adjacent detectors in a module are daisy chained so that the total number of analog channels of VDET91 is 73,728 .

Each 'module' is produced by glueing together two different ceramic substrates housing the front-end electronics for reading-out the two sides. The ceramics are actually very sophisticated thick film hybrid circuits designed to operate detectors and electronics and to supply steering and control signals [6]. To reduce the amount of multiple scattering the thickness of these ceramics has been reduced to $250 \mu \mathrm{m}$.
The silicon detectors are directly glued on the back of the ceramic substrate housing the $z$ electronics. On this side a thin layer of gold prevents interference between the $r-\phi$ and $z$ electronics and a set of spacers provides insular tion between the gold layer and the silicon detectors. The assembly procedure of the microstrip detectors has been carefully studied to guarantee a strip parallelism within $\pm 5 \mu \mathrm{m}$, a maximum deviation from the nominal position of each strip in the plane within $\pm 7 \mu \mathrm{m}$ and a planarity in a module within $\pm 15 \mu \mathrm{m}$.

Two such modules, equipped with flexible kapton connectors, are coupled together by glueing two ceramic ribs on the $z$-side ceramics and form a 'face'.

\section{The Electronics}

The thick film hybrid circuits of each 'module' house three types of custom designed CMOS chips: a charge sensitive amplifier (CAMEX64A), a steering chip and a line driver[6].

The CAMEX64A is a VLSI chip with 64 charge sensitive amplifiers on a $100 \mu \mathrm{m}$ pitch [7]. It provides signal amplification, noise filtering, parallel storage and serial read-out. Switched capacitor filters are used to reduce the noise. Using four-fold double correlated sampling a very good noise performance is achieved $\mathrm{ENC}$ (electrons) $=335+$ $30 \mathrm{C}[\mathrm{pF}]$ under optimal conditions. To minimize the need of cooling it was decided to operate the front-end electronics in conditions of reduced power consumption (below $1.0 \mathrm{~mW}$ per channel) accepting a slightly increased noise. Four of these chips are daisy-chained multiplexing 256 channels on one signal line which appears as a sequence of 256 analog voltage levels. The entire information of each detector'module' is therefore carried by six of these output signals. The detectors are AC coupled to the preamplifiers via a set of small capacitors on quartz substrate [4].

The two other custom designed chips are a steering chip which generates the sequence of digital signals needed to read-out the CAMEX64A and a line driver which drives the differential outputs into a $50 \Omega$ twisted pair cable.

The output signals are routed to several SIROCCO IV modules [8]. This unit is a 10 -bit flash ADC with a powerful DSP which allows sophisticated pedestal calibration, zero suppression, subtraction of 'common mode' noise and online cluster finding. An online threshold is used to identify the fired strips which act as cluster 'seeds'. To reduce the amount of information on tape only 7 strips on each side of a cluster 'seed' are digitized.

\section{The Mechanical Structure}

The mechanical structure of VDET91 is shown in fig. 2 . The 24 'faces' are mounted on the two inner rings.

A very light structure connects the two frames and guarantees stiffness and mechanical stability. The two outer rings, connected by light tubes, are used to fix all signal and service cables. The two sets of rings (inner and 
outer) are left mechanically independent in order to pro tect the fragile detector elements from stresses produced by the cables. The whole structure, including connecting tubes, has been produced in carbon fibre. Small aluminum inserts embedded in the fibre have been precisely machined to house screws and precision reference pins. A composite structure ( $30 \mu \mathrm{m}$ thick plastic foil coupled to $25 \mu \mathrm{m}$ aluminum and glued to carbon fibre $125 \mu \mathrm{m}$ thick) is also used for inner and outer covers as electrical and mechanical shielding.
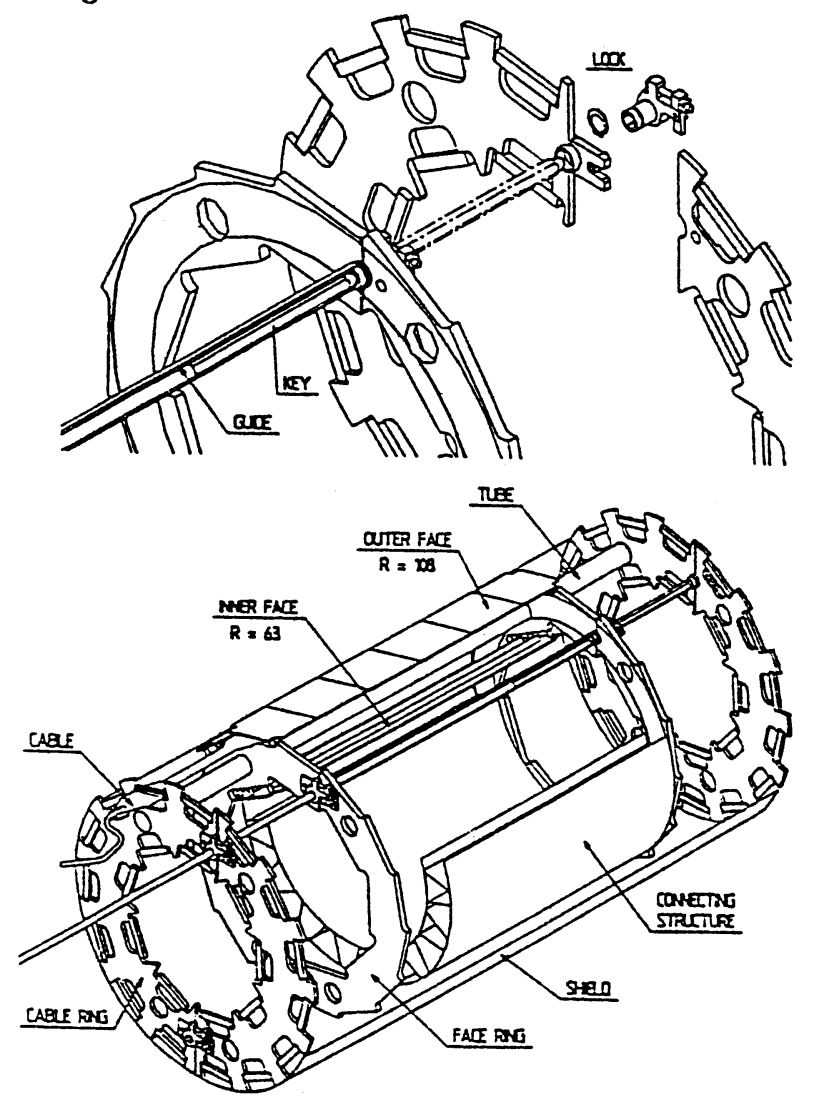

Fig.2 The mechanical structure of VDET91

The whole structure is divided in two parts to allow the installation inside Aleph with the beam-pipe already in place. The two sectors fully equipped with detectors are assembled independently. A mounting jig positioned around the beam pipe allows the coupling of the two parts in the core of Aleph. The vertex detector sits between the beam pipe and the Inner Tracking Chamber (ITC, a drift chamber with an inner radius of $12.8 \mathrm{~cm}$ ). VDET91 is supported by two rails produced on the carbon fibre wall of the ITC. The two rails are also used to guide the entire detector into the middle of the interaction region. A system of cables allows the insertion (and removal) of the detector into its final position to match a positioning pin.

The total weight of the complete detector, excluding cables, is below $2 \mathrm{Kg}$ and the overall material corresponds to about $4 \%$ of radiation length at $90^{\circ}$. The heat produced by electronics $(\sim 60 \mathrm{~W})$ is removed by blowing cool air in the whole central region of Aleph.

\section{RUNNiNg Experience}

Since April 1991 VDET has been taking data in Aleph. If we exclude the very first part of the run spent in debugging the new DAQ system and in setting-up the best operational conditions of the new apparatus, the overall data taking efficiency was fairly high $(\varepsilon \geq 86 \%)$, corresponding to 200,000 hadronic $Z^{0}$ 's collected so far.

The entire period was quite smooth apart from a few electronics failures and some beam losses which have damaged a small fraction of channels. The beam accidents were related to circumstances in which the beams circulating in LEP were suddendly lost apparently into our apparatus[1]. The total number of channels affected by these accidents were $\sim 5 \%$ of the total.

The cooling system worked properly. Temperature sensors measure a few degrees $\mathrm{C}$ difference between inlet and outlet air and a good overall stability with time $\left(\leq 1^{0} \mathrm{C}\right)$.

\section{Performance and Results}

The analysis of the collected events allows a careful study of the performance of the detector in a real collider environment. In order to use the Vertex Detector for track reconstruction its position has to be known with a prec:sion comparable to its intrinsic resolution.

Before installation, the detector was measured with an optical measuring device, but there are many possible differences between the environment of the measuring shop and the real final position of the detector (thermal effects, gravity, various stresses and constraints), and an in-situ alignment was required. The final position of each wafer is therefore measured using tracks and considering the discrepancy between hits and extrapolated impact points of the tracks as reconstructed by the central tracking system of Aleph (ITC and TPC). The full alignment procedure is described in detail elsewhere [9].

Using about 10,000 hadronic events the final position of each strip of VDET can be known with a precision better than $7 \mu \mathrm{m}$ in the wafer plane and $15 \mu \mathrm{m}$ in the perpendicular direction. Monte Carlo studies indicate that with these uncertainties we expect at most a $5 \%$ degradation of impact parameter resolution for high momentum particles.

Signal-to-noise ratio in double-sided silicon has been studied as a preliminary check for online thresholds and to tune the parameters of the offline clustering algorithm. The average r.m.s. noise width is $34 \mathrm{ADC}$ counts. About $4 \%$ of the analog channels are dead, saturated or too noisy. The signal comes from the sum of the pulse heigths in clusters associated to charged tracks [fig.3]. An offline cluster algorithm is applied to the online clusters to identify the strips that truly fired. A cluster is kept only when its position is compatible with the expected impact point of the corresponding charged track. On average only 2-3 strips contribute to real clusters. The peak of m.i.p. is 
$\sim 500 \mathrm{ADC}$ counts both for $\phi$ and $z$ sides leading to a $\mathrm{S} / \mathrm{N}$ $\sim 15: 1$. Under these conditions it has been possible to run the detector at very low occupancy: on average $\sim 15$ hits on the $r-\phi$ and $\sim 15$ hits on the $z$ side.

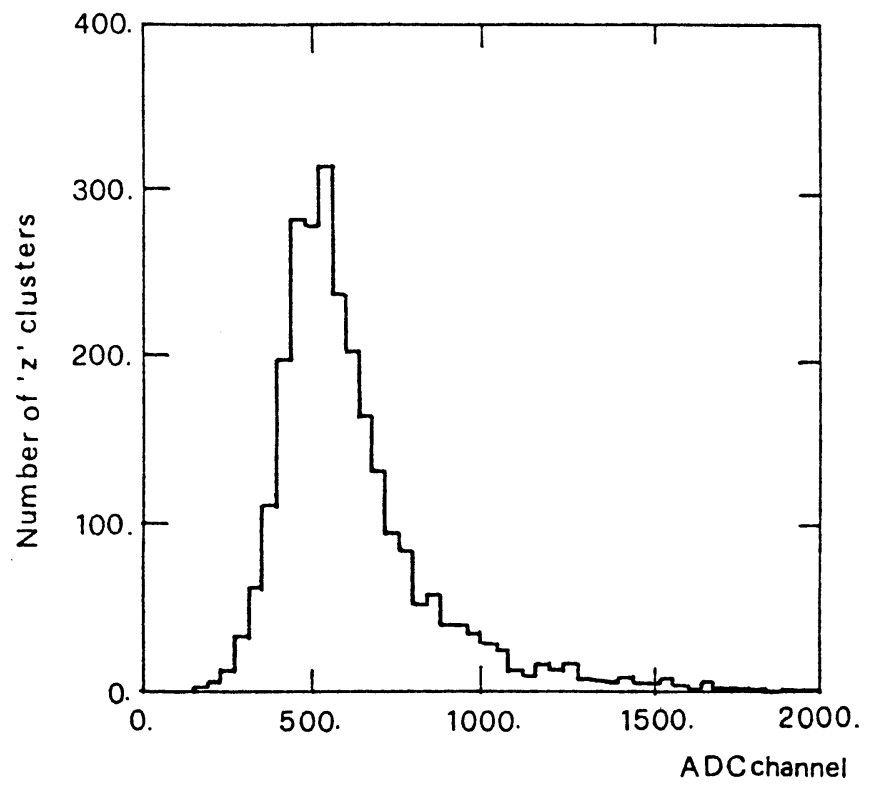

Fig.3 Signal distribution for $z$-side

Detector efficiency has been studied using $q \bar{q}$ events and plotting for each wafer the fraction of hits masching tracks over the total number of tracks passing through the angular region covered by VDET. This number accounts not only for dead strips or saturated electronics channels but also for possible inefficiencies in clustering algorithms and track matching routines that are under study. Nevertheless, for the moment we obtain average efficiency per layer/per view ranging between 91 and $93 \%$ including the few bad regions damaged by the beam accidents which have been previously described.

The intrinsic spatial resolution of VDET91 has been studied using high momentum muons passing through the wafer azimuthal overlap region. Muon tracks are constrained to pass through one of the two VDET hits. The residual distribution between the other hit and the expected impact points extrapolated by the track can be used as a measurement of the detector resolution. The width of this distribution is $\sqrt{ } 2$ times the detector accuracy. The results are shown in fig.4. From a gaussian fit we obtain a single hit resolution of $12 \mu \mathrm{m}$ in $r-\phi$ and $13 \mu \mathrm{m}$ in $z$.The resolution in $z$ has been measured considering only tracks with perpendicular incidence.

These values are in good agreement with the resolution measured in test beam and with the performance expected from general considerations on read-out pitch and signalto-noise ratio.

Using muons pairs it is also possible to quote a $30 \% \mathrm{im}$ provement in momentum resolution for stiff tracks. Fig.5 shows the distribution of the ratio $E_{(\text {beam })} / p_{(\text {muon })}$ for dimuon events when all information of the Aleph track- ing system is used ( TPC-ITC-VDET). From the width of this distribution we obtain $\frac{\Delta p}{p}=6.6 \times 10^{-4} p$ to be compared with a previous result of Aleph without VDET $\frac{\Delta p}{p}=8.8 \times 10^{-4} p$.

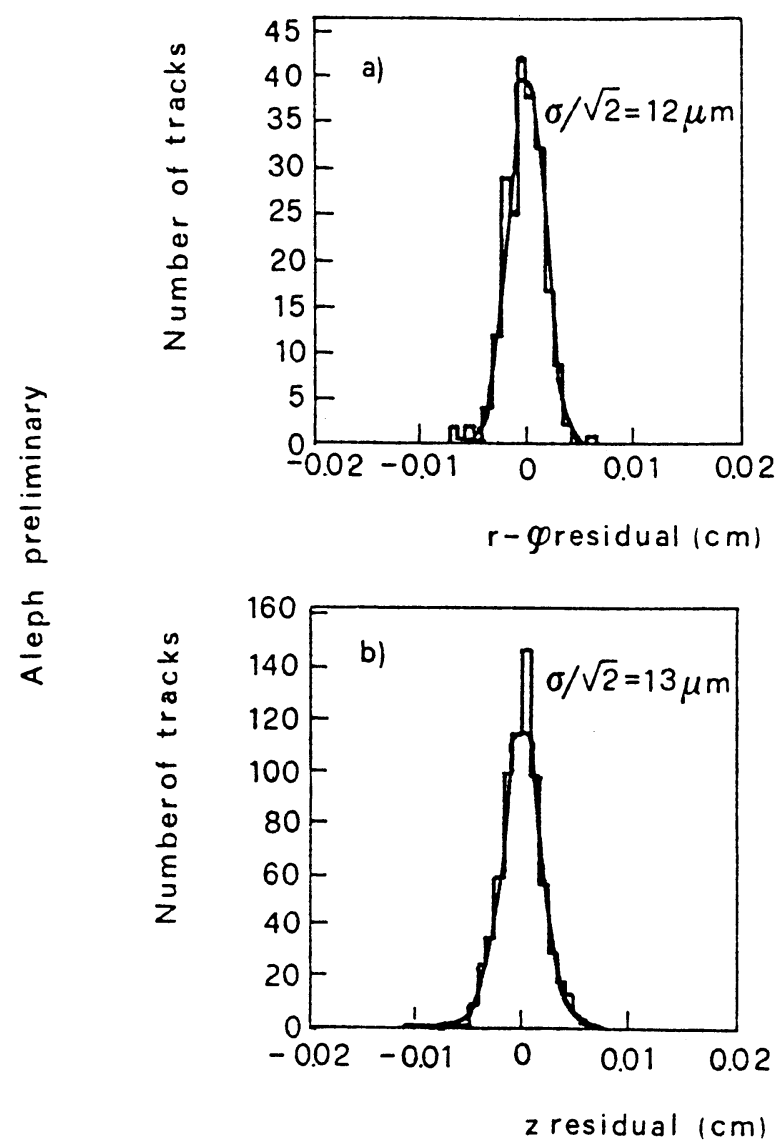

Fig.4 Single hit resolution in $r-\phi$ and $z$ projections

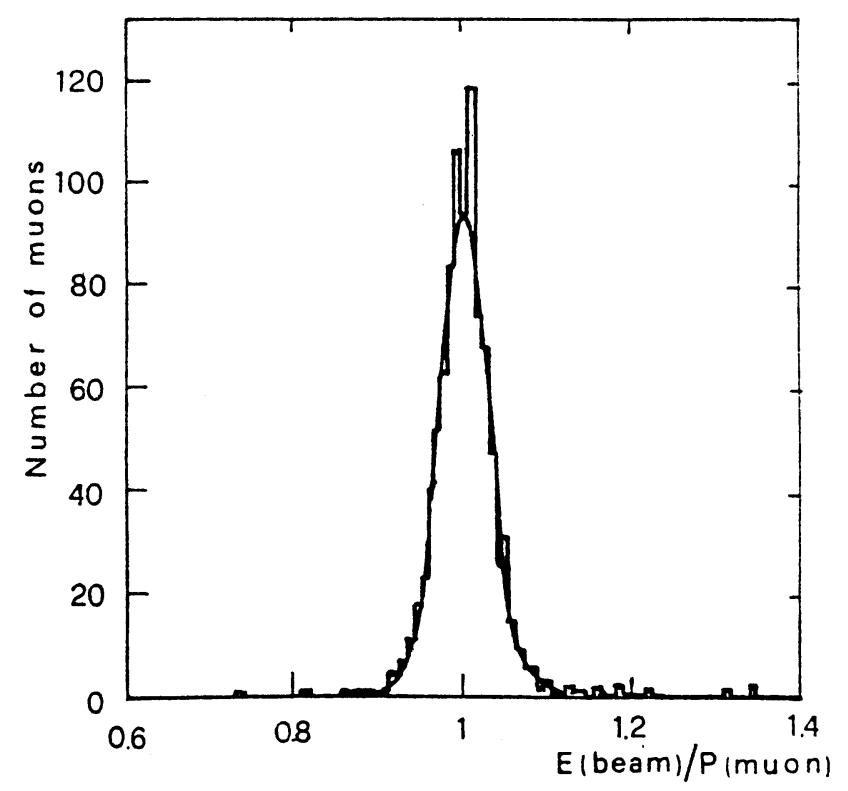

Fig.5 Momentum resolution measured using high momentum muons 
The real figure of merit of a vertex detector is, of course, the impact parameter resolution and the ability of reconstructing primary and secondary vertices.

The performance in impact parameter resolution has been studied using as a variable the muon pairs ' miss distance' measured as the sum of impact parameters of high momentum muons. This variable includes contributions from the point resolution of VDET, alignment errors and performance of the other tracking devices particularly in the measurement of the track direction.

The width of the distribution of the muons 'miss distance ' is $\sqrt{ } 2$ times the impact parameter resolution. From a gaussian fit to the distributions shown in fig. 6 we obtain a preliminary impact parameter resolution of $26 \mu \mathrm{m}$ in $r-\phi$ and $34 \mu \mathrm{m}$ in the $z$ projection. The larger value in $z$ comes mainly from lack of $z$ information in the ITC and larger systematics errors in the TPC $z$-view due to uncertainties in drift velocity.

The improvement produced by VDET in this field is well shown by the comparison between the actual values of impact parameter resolution in the two projections and the corresponding values obtained with TPC and ITC only: $107 \mu \mathrm{m}$ in $r-\phi$ and $915 \mu \mathrm{m}$ in $z$.

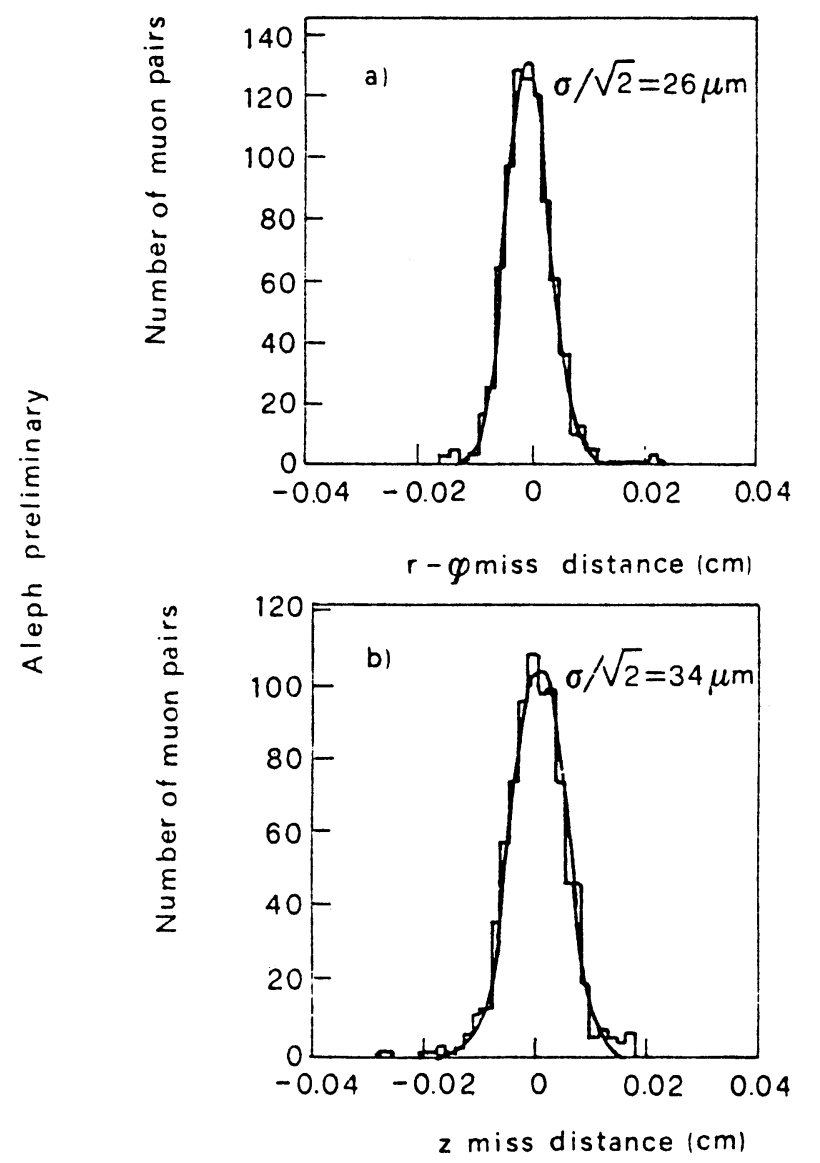

Fig. 6 Impact parameter resolution in $r-\phi$ and $z$ projections.

With such a good accuracy we are very sensitive to the beam position and we can measure very precisely the centroid and the width of the beam envelope.
Fig.7 shows a scatter plot of impact parameter and azimuthal angle distribution for leptons constrained to the beam energy. in different fills. The impact parameter is measured with respect to the reconstructed beam centroid. From this plot we can perform a simple fit to the beam size by fitting the quantity: $\sigma_{D_{0}}^{2}=\left(\sigma_{x} \sin \phi_{0}\right)^{2}+\left(\sigma_{y} \cos \phi_{0}\right)^{2}$ where $\sigma_{x}, \sigma_{y}$ are the widths of the beam envelope in the transverse plane. As result of this fit we obtain $\sigma_{x}=$ $149 \pm 3 \mu \mathrm{m}$ and $\sigma_{y}=19 \pm 3 \mu \mathrm{m}$.

The very high accuracy of VDET allows the measurement of the beam centroid with an accuracy of $7 \mu \mathrm{m}$ with 100 hadronic events only. Independent measurements show that we are able to follow the beam movements during the same fill and provide an absolute calibration of other very sensitive detectors installed by the LEP division inside the accelerator (namely the Beam Orbit Monitors, BOMs [10]).

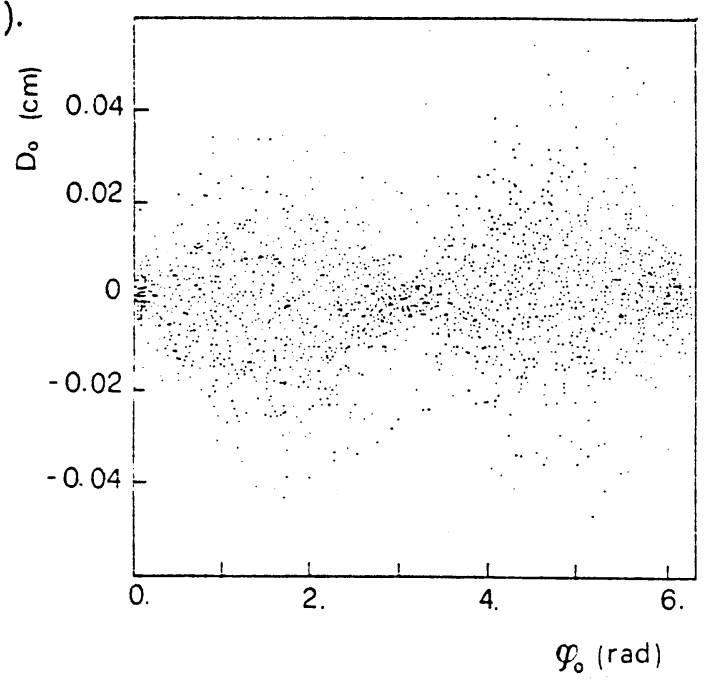

Fig.7 Scatter plot of impact parameter and azimuthal angle distribution for leptons in various fills.

\section{VDET91 READY FOR PHYSICS}

The encouraging performance of VDET 91 seem very helpful in improving the precision of the standard analysis techniques. A typical example is the quality of the lepton impact parameter distribution which is used to extract the b-lifetime information. Fig. 8 shows this distribution with and without VDET information. In fig. $8 \mathrm{a}$ ) the lepton impact parameter is measured with respect to the centroid of the beam spot while in fig. 8 b) the tracks have been refitted using the hits of VDET and the production vertex position has been reconstructed on an event-by-event basis.

The possibility of reconstructing secondary vertices is very powerful in reducing combinatorial background when looking for heavy flavoured mesons in invariant mass distributions. As an example we can show the search for $D^{0} \longrightarrow K \pi \pi \pi$ in hadronic events. With the requests of track momentum greater than $2 \mathrm{GeV} / \mathrm{c}$ and $K$ identification using $\frac{d E}{d x}$ when available from the TPC, the signal appears as in fig. 9 a). The same simple analysis with the 
additional request of a secondary vertex and a $\chi^{2}$ cut on the vertex itself yields the result shown in fig. 9 b). Here we select events in which the decay length of the $D^{0}$ candidate is greater than $800 \mu \mathrm{m}$ in the transverse plane with respect to the average beam position.

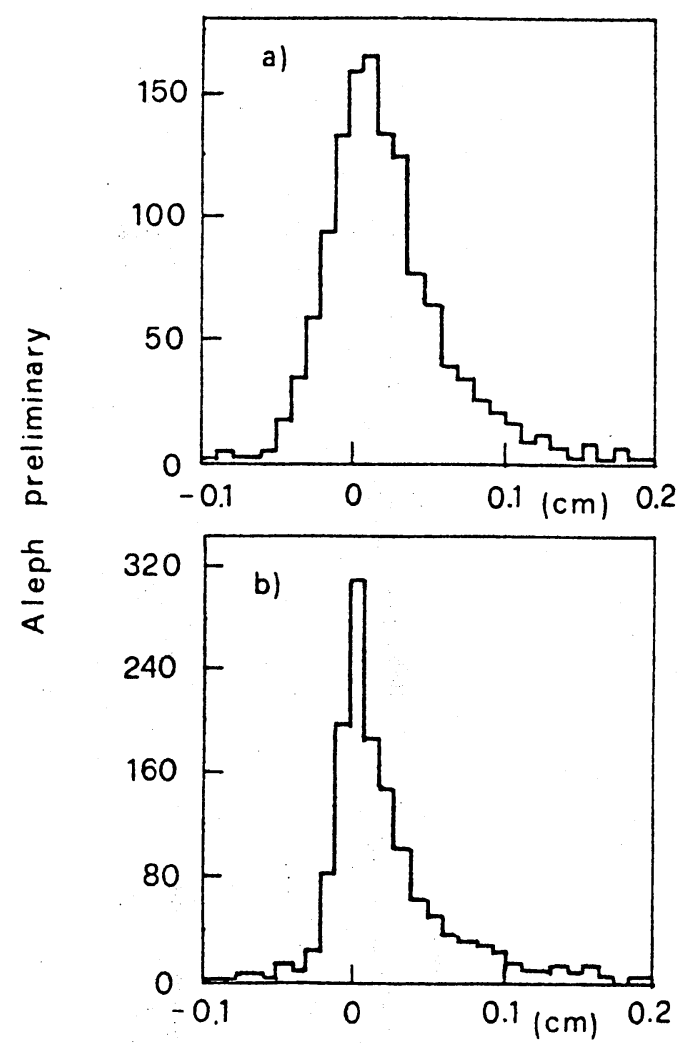

Fig.8 High $p_{t}$ leptons impact parameter distribution a)without and b)with VDET information

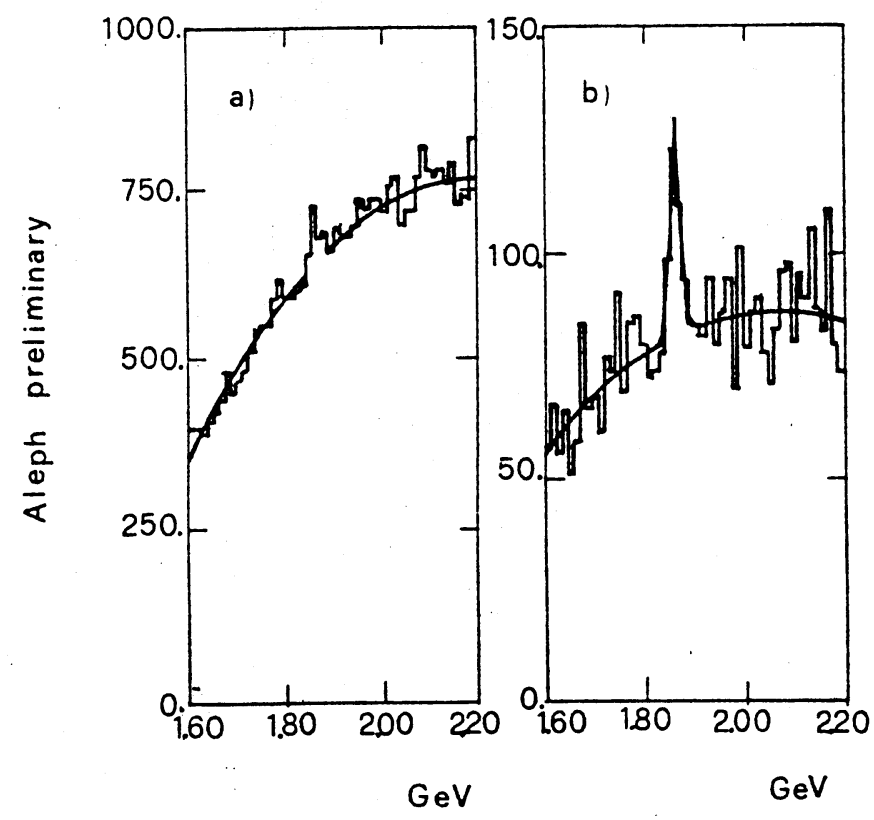

Fig.9 Invariant mass distribution of $D^{0} \rightarrow K \pi \pi \pi$ candidates a)without and b)with request of a secondary vertex

\section{Conclusion}

A complete detector with two concentric layers of microstrip silicon detectors incorporating double side readout has been taking data at CERN $e^{+} e^{-}$LEP collider since April 1991. Preliminary results on single hit and impact parameter resolution show that the detectors and VLSI read-out electronics work as expected and the performance compare well to previous test beam results and Monte Carlo simulations. Preliminary results on precision vertex reconstruction show a significant improvement in the data analysis which will be fully exploited in studying $\tau, \mathrm{b}$ and $\mathrm{c}$ physics when all statistics are available.

\section{REFERENCES}

[1] G. Batignani et al., Nucl. Phys. B (Proc. Suppl) 23A (1991) 291

[2] D. Decamp et al., Nucl. Instr.\& Meth. A294 (1990) 121

[3] A comprehensive report on the design, construction and use of the device is in preparation.

[4] G. Bagliesi et al., INFN/PI/AE 86/10

G.Batignani et al. Nucl. Instr. $\mathcal{O}$ Meth A273(1988) 677

G. Batignani et al., Nucl. Phys. B (Proc. Suppl) 23A (1991) 297

G. Batignani et al., Proceedings of the 5-th Pisa Meeting on Advanced Detectors To be published in Nucl. Instr.\& Meth. (1991)

[5] J. Sedlmeir Thesis, Technische Universität, Munchen 1985.

G. Lutz, MPI-PAE/Exp. El.175 and "Vertex

Detectors", Plenum Press, NY (1988) 195

P. Holl et al., IEEE Trans. Nucl.Sci. 36

(1988) 251

[6] H. Becker et al., IEEE Trans. Nucl. Sci. 36 (1988) 246

[7] W. Buttler et al., Nucl. Instr. \& Meth. B279 (1989) 204

[8] N. Bingefors et al., DELPHI Internal Note 88-48 (1988)

[9] D. Brown et al., ALEPH Internal Note 91-43 (1991)

[10] W. Kozanecki et al., ALEPH Internal Note 91-47 (1991) 